\title{
Herbal medicine (Danggui-Shaoyao-San) and Ear Acupoint Pressing Beans in the treatment of dysmenorrhea caused by endometriosis and adenomyosis: a study protocol randomized controlled trial
}

\section{Yingzhou Tian}

Guangdong Hospital of Traditional Chinese Medicine

Jiayi Wu

Guangdong Hospital of Traditional Chinese Medicine https://orcid.org/0000-0003-3933-8159

\section{Yanxiong Huang}

Guangzhou University of Chinese Medicine

\section{Xuefang Liang}

Guangdong Hospital of Traditional Chinese Medicine Lixing Cao ( $\sim$ lixingcao@126.com)

Study protocol

Keywords: Danggui Shaoyao San, Ear pressing beans, Endometriosis, Adenomyosis, Dysmenorrhea, Randomized controlled trial

Posted Date: May 31st, 2019

DOI: https://doi.org/10.21203/rs.2.9951/v1

License: (c) (1) This work is licensed under a Creative Commons Attribution 4.0 International License.

Read Full License 


\section{Abstract}

Endometriosis and adenomyosis are two of the most common causes of secondary dysmenorrhea and often lead to a deterioration in the quality of life. Traditional Chinese medicine and acupuncture are widely used in the treatment of menstrual pain in clinical practice. Danggui Shaoyao San (DSS) and ear acupoint pressing beans may constitute an effective treatment strategy for women with dysmenorrhea due to endometriosis and/or adenomyosis, although evidence is limited. Methods/design This randomized, controlled clinical trial aims to recruit patients who suffer from menstrual pain due to endometriosis and/or adenomyosis to evaluate the efficacy and safety of DSS and auriculotherapy. Primary outcome measures are Visual Analog Scale (VAS), Short-Form McGill Pain Questionnaire (SFMPQ), dysmenorrhea symptoms and traditional Chinese medicine correlative time points. Discussion This pivotal trial will be a standardized, scientific, clinical trial designed to evaluate the use of DSS and auriculotherapy in the treatment of dysmenorrhea due to endometriosis and/or adenomyosis. The trial will also conform to the international standards of clinical trials for the recognition of traditional Chinese medicine. Trail registration Chinese Clinical Trail Registry, ID: ChiCTR-IOR-17013829 Registered on 11th December 2017 Keywords: Danggui Shaoyao San; Ear pressing beans; Endometriosis; Adenomyosis; Dysmenorrhea; Randomized controlled trial

\section{Background}

Endometriosis and adenomyosis are two of the most common causes of secondary dysmenorrhea. Endometriosis is found in $70 \%-90 \%$ of patients complaining of pelvic pain (1-3) while adenomyosis is considered to be a subtype of endometriosis that is confined to the uterine wall. Both conditions may cause dysmenorrhea and infertility.

First-line treatment options for pain due to endometriosis include nonsteroidal anti-inflammatory drugs (NSAIDs), oral contraceptives (OC) and progestogens (4). Hormone therapy, such as gonadotropinreleasing hormone agonist ( $\mathrm{GnRHa}$ ) and the levonorgestrel-releasing intrauterine system (LNS-IUS), is widely used in the treatment of adenomyosis and endometriosis $(5,6)$. However, the high recurrence rate, side effects and severe adverse events associated with these treatments lead patients to seek supportive complementary and alternative forms of medicine (CAM) (7). Examples of such CAM therapies for dysmenorrhea include acupuncture-related therapies (8-11), as well as Chinese herbal medicine (CHM) (12-18). Danggui-Shaoyao-San (DSS), also known as Toki-Shakuyaku-San or TJ-23, is a widely used traditional formulation of Chinese medicine (TCM) derived from "Jin Kui Yao Lue", first described by Zhong-Jing Zhang in the Eastern Han Dynasty. DSS consists of six Chinese herbs: Radix Paeoniae Alba, Radix Angelica sinensis, Rhizoma Chuanxiong, Poria cocos, Rhizoma Atractylodis macrocephalae, and Rhizoma Alismatis. For thousands of years, DSS has been used as a blood-activating and stasiseliminating drug to treat gynecological disorders, including dysmenorrhea, amenorrhea and infertility $(19,20)$. DSS is believed to mediate ovarian hormones (21), estrogenic activity (22) and neurotoxicity (23). Auricolotherapy, or auricular point therapy (APT), has been found to relieve menstrual pain in patients with dysmenorrhea and endometriosis (24-27). However, the application of this treatment modality 
currently doesn't meet the Good Clinical Practice standards. In order to assess and add the proper application of TCM and Ear Acupoint Pressing Beans, precise studies are now needed to confirm the safe and effective use of Chinese herbal medicines and APT for the treatment of dysmenorrhea caused by endometriosis and adenomyosis .

Here, we describe a protocol for a randomized, controlled clinical trial to evaluate the safety and effectivity of the use of DSS and APT in the treatment of gynecology conditions.

\section{Methods/design}

\subsection{Study design}

This study is a randomized, controlled, clinical pilot trial. We will select patients who experienced dysmenorrhea caused by endometriosis and adenomyosis between November 2017 and December 2019 at Guangdong Provincial Hospital of Chinese Medicine. Patients will be randomly assigned to the following groups: (1) Danggui Shaoyao San (DSS) group; (2) Danggui Shaoyao San (DSS) and auricular point therapy (APT) group and (3) oral contraceptives (OC) group. This will be carried out according to stratified random sampling and each patient will be allocated a random number (corresponding to numbered drugs). We will then evaluate the efficacy and safety of Danggui Shaoyao San (DSS), and ear acupoint pressing beans, for patients by comparing a range of indices between different groups, including the relief of pain, serum CA 125 level, hepatorenal function, coagulation function and electrocardiograms. The study protocol will be certified by the Institutional Review Board (IRB) of The Second Affiliated Hospital of Guangzhou University of Chinese Medicine (Guangdong Provincial Hospital of Chinese Medicine), and registered at the Chinese Clinical Trial Registry (http://www.chictr.org.cn/, ChiCTR-IOR-17013829).

The below inclusion criteria, exclusion criteria, and elimination criteria will be followed to ensure the accuracy of results.

\subsection{Inclusion criteria}

1) Patients with dysmenorrhea caused by endometriosis or/and adenomyosis

2) Traditional chinese medicine syndrome, blood deficiency and blood stasis

3) Aged 18-42 years, Non-menopausal females with a regular menstrual cycle (23-37 days)

4) Provision of signed and informed consent

\subsection{Exclusion criteria}

1) Surgical treatment of patients in the near future 
2) Patients with other severe gynecological conditions, including pelvic infection and gynecological malignant tumor(s)

3) Cardiovascular, liver, kidney, brain, lung co-morbidity, or other serious disease

4) Metal disability

5) Serum CA $125 \varangle 200 \mathrm{U} / \mathrm{ml}$

6) Ovarian endometrioma maximum anteroposterior diameter $₫ 5 \mathrm{~cm}$, or other ovarian cystic-solid/solid mass maximum anteroposterior diameter $₫ 5 \mathrm{~cm}$

7) Uterine leiomyomas with a maximum steroposterior diameter $₫ 5 \mathrm{~cm}$

8) Use of hormones, or other medications, including GnRH-a, during the past 3 months

9) Secondary dysmenorrhea cause by other diseases, except for endometriosis and andenomyosis

10) Patients who plan to become pregnant in 6 months.

\subsection{Study withdrawal and stopping medication}

1) Serious complications, or requiring other treatments during the study

2) Other complications arising due to interventions, including severe allergy or serious adverse events

3) A worsening condition that cannot be controlled

4) Refusal to continue the treatment, regardless of the reason

This part mainly depend on LXC, XFL and other experienced gynecology professor.

\subsection{Trial procedure}

The entire trial includes 3-menstrual cycle intervention phases and a 3-menstrual cycle follow-up phase. Participants will be randomized to receive one of three treatments (DSS, DSS in combination with APT (DSS+APT), or OC therapy) through a stratified randomization system, and acquire a random number (corresponding to the DSS group, DSS+APT group and OC group). Subjects in the DSS and DSS+APT groups will be instructed to apply the DSS decoction twice every day, beginning two weeks prior to the menstrual period for 21 days. Subjects in the DSS+APT group will undergo APT twice a week during the three weeks they take DSS. Subjects in the OC group will take one tablet daily, beginning on day five of their menstrual cycle, and continuing for 21 days. Subjects will be evaluated once a month and results recorded in a Case Report Form (CRF) until the study is completed. A participant flowchart is shown in Fig. 1 and the Standard Protocol Items will be as follows. A 'Recommendations for Interventional Trials 
(SPIRIT)' Figure for the trial is shown in Table 1. Adverse events will be monitored during face-to-face and telephone interviews throughout the study.For participants, during this six-month, relatived medicine or treatment are banned.

\subsection{Interventions}

The oral DSS decoction used in the study will be produced by the TCM pharmacy of Guangdong Provincial Hospital of Chinese Medicine, which holds a Good Manufacturing Practice certificate. Both DSS and DSS+APT group subjects will be provided with individually packaged doses of DSS decoction, with instructions stating that each dose should be heated in warm water and consumed twice daily, beginning two weeks prior to menstruation, for a total duration of three weeks.

All acupuncturists with TCM certification will receive specific practical training prior to the study. APT group subjects will undergo APT twice a week, beginning two weeks prior to menstruation and lasting a total of three weeks. The set of auricular points will include Ear-Shenmen (MA-TF1), Sympathetic (AH6a), Uterus (TF2), Subcortex (AT4), and Kidney (MA-SC). One auricle will be sterilized with $75 \%$ alcohol and the supra-auricular points will be treated with Wang Bu Liu Xing (cowherb seeds) in desensitized plasters $(5 \times 5 \mathrm{~mm})$. Ears will be alternated every three days.

Subjects in the OC group will take one tablet daily, starting on day five of their menstrual cycle, and continuing for 21 days.

\subsection{Randomization}

The study leader(LXC and LXF) will create random sequences using envelopes. Randomization will take place as soon as subject eligibility requirements are met. Participants will be randomized into one of the three treatment groups (DSS, DSS+APT, or OC). Random numbers will be generated by using a special website, http://www.gztcmgcp.com/sjxt/login.asp and saved by statistical professionals.

\subsection{Quality control}

To ensure that this research will be good quality, this study protocol has undergone repeatedly amendment and alteration by gynecological disease specialists, skilled acupuncturists, professional statisticians and experienced methodologists. The entire study routine will be operated by independent qualified inspectors. The CRF will be filled out under the guidance of the CRF instructions. Original medical history and data recorded in the CRF, will not be modified afterwards. Any record alteration must be mark in detail and accompanied by a signature of the individual making any changes. All laboratory and clinical data is to be recorded rigorously, and all significantly abnormal data will be reappraise. Physicians will be required to make any necessary notes in response to such information.

\subsection{Outcome measures}


Our primary outcome measures are as follows: Visual Analog Scale (VAS) for pain, Short-Form McGill Pain Questionnaire (SF-MPQ), Dysmenorrhea symptom timepoints and TCM correlative symptom timepoints.

Our secondary outcome measures include pelvic examination, serum CA125, T lymphocyte subset levels, B lymphocyte subset levels, natural killer (NK) cell levels in human peripheral blood mononuclear cells (PBMCs), transvaginal/transrectal ultrasound (volume of the uterus and ovary, maximum anteroposterior diameter of the ovarian endometrioma).

\subsection{Safety assessments}

Routine blood tests, liver and kidney function tests, and an electrocardiogram will be performed both before and after treatment. Any adverse events will be observed and recorded in detail at any time during the treatment phase, as well as the follow-up phase. Measures will be taken in time when adverse events happens.

\subsection{Estimation of sample size}

Our sample size estimation is based upon previous prospective clinical studies (28). The efficacy of DSS is assumed to be $95 \%$. However, due to the lack of existing data relating to the efficacy of APT, we cannot rely upon the literature for sample size determination. Instead, we hypothesized that the order of efficacy for the treatments will be as follows: DSS+APT $\triangle D$ SS = OC. PEMS 3.1 (Package for Encyclopaedia of Medical Statistics, Si chuan, China)software for Windows was used to randomly assign patients to one of three treatment groups. The rounded sample size for each group was 54 . Assuming a $10 \%$ dropout rate, the final sample size would be 60 for each group. Therefore, 180 participants will be recruited. Guangdong Provincial Hospital of Chinese Medicine, which owns large number of outpatients, and experienced project directors ensure the trials progress.

\subsection{Data collection and statistical analyses}

Data will be collected and recorded in CRF forms via doctors' office visiting and telephone follow-ups; this will occur once every menstrual period. The Epidata 3.1 Statistical Package will be used for data input. Two people will perform data entry independently; these people will be trained before and tested prior to starting work. The data will then be analyzed using the Statistical Package for the Social Sciences (SPSS) software (IBM, Armonk, NY, USA.)

The data will be analyzed by an independent statistician.For measurement data, the mean, standard deviation(SD), minimum value, maximum value, and median will be used for comparison.

Differences among groups in categorical data will be analyzed by analysis of variance (ANOVA) and pairwise comparison, while heteroscedasticity data or abnormal distribution will be analyzed by the rank-sum 
test and pair-wise comparison. If the data are divided as count data, the constituent ratio and frequency will be appropriate for comparison. The chi-square test at an $a=0.05$ given a 2 -sided test will be suitable for analysing efficient intergroup difference.

\section{Discussion}

Endometriosis and adenomyosis are estrogen-dependent conditions, which result in dysmenorrhea (i.e. menstrual and chronic pain), which occurs in young to middle-aged adult women. A variety of treatments are used by clinicians to reduce such pain, including surgery, NSAID, OC, progestogen, danazol, GnRH-a, and anti-progestogen medications (29). Acupuncture is an adjunctive therapy for pelvic pain associated with endometriosis. Two randomized studies have previously evaluated specific versus sham acupuncture for endometriosis pain and both reported significantly better pain relief with true acupuncture $(30,31)$. In addition, previous randomized clinical trials, comparing Chinese herbal medicine treatment to gestrinone and danazol, concluded that Chinese herbal medicine had comparable results, but with fewer side effects (32). Taking into consideration the TCM "same treatment for different diseases and different treatments for the same disease" therapeutic principle, we designed this study to evaluate the efficacy and safety of Chinese herbal medicine DSS and APT for the treatment of menstrual pain due to endometriosis and/or adenomyosis, and to provide a scientific basis/rationale for the clinical application of these two treatments.

This study was designed to evaluate the efficacy and safety of Danggui Shaoyao San (DSS), and ear acupoint pressing beans, for the treatment of dysmenorrhea associated with endometriosis and adenomyosis. We also wished to provide a scientific basis for therapy efficacy.

\section{List Of Abbreviations:}

APT: Auricular points therapy

CAM: Complementary and alternative medicine

CHM: Chinese herbal medicine

CONSORT: Consolidated Standards of Reporting Trials

CRF: Case Report Form

DSS: Dangggui Shaoyao San

GCP: Good Clinical Practice

ITT: Intention-to-treat

LOCF: Last observation carried forward 
NSAIDs: Nonsteroidal anti-inflammatory drug

OC: Oral contraceptives

SF-MPQ: Short-Form McGill Pain Questionnaire

SPIRIT: Standard Protocol Items: Recommendations for Interventional Trials

SPSS: Statistical Package for the Social Science

TCM: Traditional Chinese Medicine

VAS: Visual Analog Scale for pain

\section{Declarations:}

\section{Ethics approval and consent to participate}

The IRB of Guangdong Provincial Hospital of Traditional Chinese Medicine approved the study protocol. (Ethical reference: B2017-158-01. Verbal informed consent will be obtained from all study participants.)

\section{Consent for publication}

Not applicable

\section{Availability of data and materials}

Not applicable

\section{Competing interests}

The authors declare that they have no competing interests.

\section{Funding}

1.The National Natural Science Foundation of China 81574008

2.Scientific special project of Guangdong Provincial Academy of Chinese Medical Sciences (2017[81]YN2016ML05)

\section{Acknowledgements}


Not applicable

Trial status: Participants are currently being recruited.

The date recruitment began at November 2017; the approximate date when recruitment will be completed:December 2019.

Hospital: Guangdong Provincial Hospital of Chinese Medicine.

Protocol version number: TRLS-D-19-00331 (Version number: 3.0)

\section{Author contributions}

XFL and LXC developed the study protocol. YZT and JYW coordinate the study. WJY and YXH is responsible for enrolment and data collection. WJY drafted the manuscript in collaboration with YZT. All authors have read and approved the final version of the manuscript.

\section{Author details}

a.Team of Application of Chinese Medicine in Perioperative Period, Guangdong Provincial Hospital of Chinese Medicine.

b.Guangzhou University of Chinese Medicine

c.Department of Gynecology, Guangdong Provincial Hospital of Chinese Medicine.

\# These authors contributed equally to this work: YingZhou Tian and JiaYi Wu.

* Address correspondence to: Lixing Cao and Xuefang Liang

Guangdong Provincial Hospital of Chinese Medicine, No. 111, Dade Road, Yuexiu District,Guangzhou 510120, China.

Telephone: 86-020-81887233-35840区

Fax: 86-020-81874903;

E-mail: YingZhou Tian: addison_tian@163.com

Jiayi Wu:wujiayi.1992@163.com

Yanxiong Huang: 1958107084@qq.com

Xuefang Liang : liangxuefang2006@126.com 


\section{Trail registration}

Chinese Clinical Trail Registry, ID: ChiCTR-IOR-17013829

Registered on 11th December 2017

\section{References}

[1] Robertz S, Antoni J D: Diagnosis of Endometriosis, SEMINARS IN REPRODUCTIVE MEDICINE, 2003, 21: 193-208.

[2] Relationship between stage, site and morphological characteristics of pelvic endometriosis and pain, Hum Reprod, 2001, 16(12): 2668-2671.

[3] Ballard K D, Seaman H E, de Vries C S, et al: Can symptomatology help in the diagnosis of endometriosis? Findings from a national case-control study-Part 1, BJOG : an international journal of obstetrics and gynaecology, 2008, 115(11): 1382-1391.

[4] Lang JH: Guideline for the diagnosis and treatment of endometriosis, Zhonghua Fu Chan Ke Za Zhi, 2015, 50(3): 161-169.

[5] Schmidt C L: Endometriosis: a reappraisal of pathogenesis and treatment, Fertility and sterility, 1985, 44(2): 157.

[6] Tsui K, Lee W, Chen C, et al: Medical treatment for adenomyosis and/or adenomyoma, Taiwanese Journal of Obstetrics and Gynecology, 2014, 53(4): 459-465.

[7] Yu A: Complementary and alternative treatments for primary dysmenorrhea in adolescents, The Nurse Practitioner, 2014, 39(11): 1-12.

[8] Chen M, Chien L, Liu C: Acupuncture or Acupressure at the Sanyinjiao (SP6) Acupoint for the Treatment of Primary Dysmenorrhea: A Meta-Analysis, Evidence-Based Complementary and Alternative Medicine, 2013, 2013: 1-8.

[9] Cho S, Hwang E: Acupressure for primary dysmenorrhoea: A systematic review, Complementary Therapies in Medicine, 2010, 18(1): 49-56.

[10] Chung Y, Chen H, Yeh M: Acupoint stimulation intervention for people with primary dysmenorrhea: Systematic review and meta-analysis of randomized trials, Complementary Therapies in Medicine, 2012, 20(5): 353-363. 
[11] Xu T, Hui L, Juan Y L, et al: Effects of moxibustion or acupoint therapy for the treatment of primary dysmenorrhea: a meta-analysis, Alternative therapies in health and medicine, 2014, 20(4): 33.

[12] X Z, M P, A B, et al: Chinese herbal medicine for primary dysmenorrhoea (Review, The Cochrane Collaboration, 2008, (2): D5288.

[13] Park K, Park K, Hwang D, et al: A Review of In Vitro and In Vivo Studies on the Efficacy of Herbal Medicines for Primary Dysmenorrhea, Evidence-Based Complementary and Alternative Medicine, 2014, 2014: 1-11.

[14] Mirabi P, Alamolhoda S H, Esmaeilzadeh S, et al: Effect of medicinal herbs on primary dysmenorrhoea- a systematic review, Iran J Pharm Res, 2014, 13(3): 757-767.

[15] Hook I L I: Danggui to Angelica sinensis root: Are potential benefits to European women lost in translation? A review, Journal of Ethnopharmacology, 2014, 152(1): 1-13.

[16] van Andel T, de Boer H J, Barnes J, et al: Medicinal plants used for menstrual disorders in Latin America, the Caribbean, sub-Saharan Africa, South and Southeast Asia and their uterine properties: A review, Journal of Ethnopharmacology, 2014, 155(2): 992-1000.

[17] Scheid V, Ward T, Cha W, et al: The treatment of menopausal symptoms by traditional East Asian medicines: Review and perspectives, Maturitas, 2010, 66(2): 111-130.

[18] Daily J W, Zhang X, Kim D S, et al: Efficacy of Ginger for Alleviating the Symptoms of Primary Dysmenorrhea: A Systematic Review and Meta-analysis of Randomized Clinical Trials, Pain Med, 2015, 16(12): 2243-2255.

[19] Jiang $H$, Shen $Y$, Wang $X$ : Current progress of Chinese medicinal treatment of endometriosis, Chinese Journal of Integrative Medicine, 2010, 16(3): 283-288.

[20] Naoki K, Tsutomu O, Ichiro S, et al: Analgesic effect of a herbal medicine for treatment of primary dysmenorrhea-a double-blind study, AMERICAN JOURNAL OF CHINESE MEDICINE, 1997, XXV(2): 205212.

[21] Usuki S, Higa T N S, Soreya K: The improvement of luteal insufficiency in fecund women by tokishakuyakusan treatment, The American journal of Chinese medicine, 2002, 30(2-3): 327.

[22] Chung M H, Suzuki S, Nishihara T, et al: Estrogenic effects of a Kampo formula, Tokishakuyakusan, in parous ovariectomized rats, Biol Pharm Bull, 2008, 31(6): 1145-1149.

[23] Lee J, Hwang D, Kim H G, et al: Dangguijakyak-san protects dopamine neurons against 1-methyl-4phenyl-1,2,3,6-tetrahydropyridine-induced neurotoxicity under postmenopausal conditions, Journal of Ethnopharmacology, 2012, 139(3): 883-888. 
[24] Cai Z: Effect of ear point therapy for treatment of primary dysmenorrhea: 91 cases observation, Gansu Medical Journal, 2010, 29(01): 55-57.

[25] Liu Z: Effect of ear point therapy for treatment of primary dysmenorrhea, Journal of Emergency in Traditional Chinese Medicine, 2006, 12(12): 1350-1351.

[26] Wu R, Zhang H, Lin L: Observation on ear point taping and pressing therapy for treatment of primary dysmenorrhea, Chinese Acupuncture \& Moxibustion, 2007, 27(11): 815-817.

[27] Li Y, Li Y, Chen S: Effect of ear point therapy and herbal medicine for treatment of endometriosis associated dysmenorrhea: 8 cases observation, Liaoning Journal of Traditional Chinese Medicine, 2002, 29(3): 144-145.

[28] Yang J: Clinical Effect of Danggui Shaoyao Powder in Treatment of Endometriosis Patients with Dysmenorrhea, Clinical Medicine \& Engineering, 2016, 23(06): 759-760.

[29] Treatment of pelvic pain associated with endometriosis: a committee opinion, Fertility and Sterility, 2014, 101(4): 927-935.

[30] Wayne P M, Kerr C E, Schnyer R N, et al: Japanese-Style Acupuncture for Endometriosis-Related Pelvic Pain in Adolescents and Young Women: Results of a Randomized Sham-Controlled Trial, Journal of Pediatric and Adolescent Gynecology, 2008, 21(5): 247-257.

[31] Rubi-Klein K, Kucera-Sliutz E, Nissel H, et al: Is acupuncture in addition to conventional medicine effective as pain treatment for endometriosis? European Journal of Obstetrics \& Gynecology and Reproductive Biology, 2010, 153(1): 90-93.

[32] Flower A, Liu J, Lewith G, et al: Chinese herbal medicine for endometriosis (Review, The Cochrane Collaboration, 2012, (5): D6568.

\section{Table}

Due to technical limitations the table could not be inserted here. It an be found as an image file in the supplemental files.

\section{Figures}




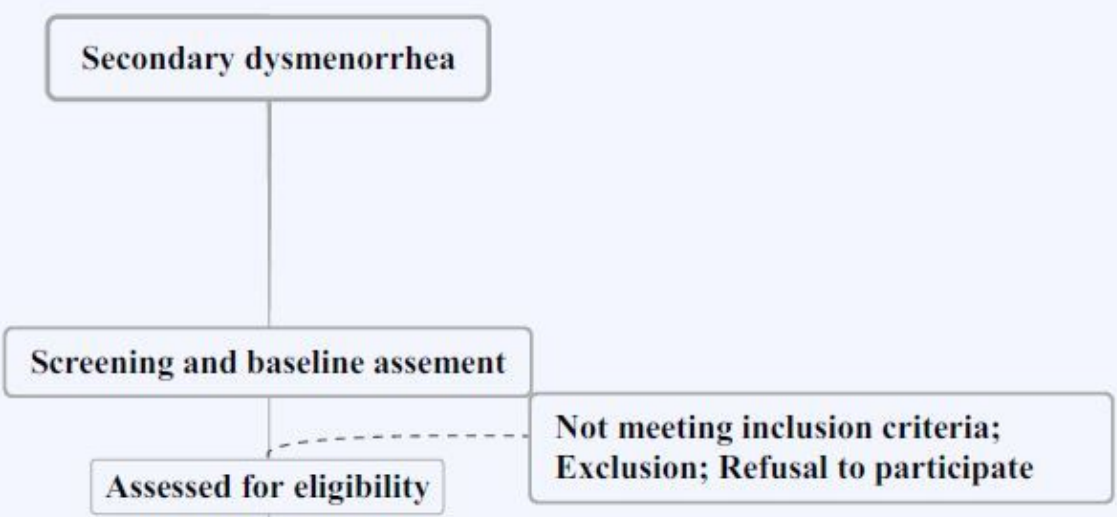

Randomized to one of three treatment groups

DSS group: Take the DSS decoction twice a day since two weeks before menstruation for three weeks
OC group: Take the one tablet everyday on day five of the menstrual cycle for three weeks
DSS+APT : DSS group + combined with auricular points therapy twice a week

Evaluate once a menstrual cylce:

VAS, SF-MGQ, dysmenorrhea symptom integral, TCm correlative symptom integral, etc. Recorded in case report form

Evaluation (three-month follow-up)

Data analysis

\section{Figure 1}

Study Flowchart.

\section{Supplementary Files}

This is a list of supplementary files associated with this preprint. Click to download.

- Table1.tiff

- InformationLeafletforInformedConsent20190410.doc

- SPIRITChecklistforrandomisedstudies20190410.doc 